\title{
Elasto-plastic analysis of the rotor and wedges supercritical turbo generator
}

\author{
Xingtian $\mathrm{Qu}^{1, \mathrm{a}}$, Yongbing Zhao ${ }^{2, \mathrm{~b}}$, Jianbing Cheng ${ }^{3, \mathrm{c}}$,Shengyu $\mathrm{Wu}^{4, \mathrm{~d}}$ and \\ Chaopeng Zhang ${ }^{5, e}$ \\ ${ }^{1,2,3,4,5}$ College of Mechanical Science and Engineering, Jilin University, Changchun, China

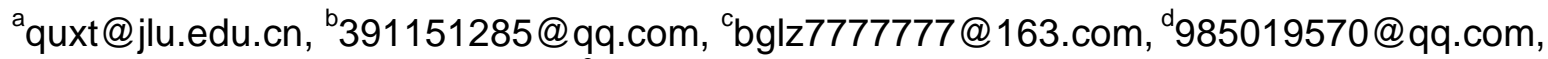 \\ e1092129913@qq.com
}

Keywords: ABAQUS; turbo generator rotor; wedges; Finite element method; Strength analysis; Elasto-plastic analysis; Stress gradient.

Abstract. Under the condition of over speed, the rotor and wedges of the supercritical 1000MW turbo generator are plastically deformed. By using the software ABAQUS for rotor and wedges strength analysis, The results showed that the strength of chamfer at the tooth root of aluminum wedges in rotor, chamfer at the end of aluminum wedge shoulder and chamfer at the sub-groove of anti-magnet part in rotor does not satisfy the requirements of the position. The actual stress distribution, plastic deformation region and maximum plastic strain of the rotor and the wedges can be obtained through elasto-plastic analysis of the rotor and the wedges. Establish several wedges and rotor finite element models with different parameters to those dangerous points that do not meet the strength requirements respectively, from which we can find the effect of each parameter on the stress of the dangerous point by comparison analysis. Different stress gradients of aluminum wedges in rotors result in a larger maximum stress value and a smaller plastic deformation region. The maximum stress value of the rotor decreased with the increase the chamfer radius. The average pressure at wedge shoulder is not related to the wedge angle. The maximum stress value of the wedge is significantly increased with the decrease of the radius of curvature at the corner, from which we can get the best design.

\section{Introduction}

The establishment of electric power industry has already had a history of more than one hundred years, and the fossil fuel-based steam thermal power generation still has more than $50 \%$, and this state will not change over a period of time[1]. In China, the production of the same amount of electricity consumes $125 \%$ coal than the international level[2]. To solve the problem of power supply coal consumption, fundamentally speaking, is to optimize the thermal power structure[3,4]. The performance supercritical generating unit is reliable, the technology is mature, and it can be adapted to the requirements of coal, so it is important to develop supercritical generating unit in China. But the large supercritical turbo generator rotor is usually about several tens or even hundreds of tons, the structure are complex, and rotate at a speed of $15003600 \mathrm{rpm}$ in the supercritical state of high temperature, high pressure and superheated steam environment. High pressure rotor is mainly under high temperature and high stress, low pressure rotor and the generator rotor are mainly under tremendous centrifugal force generated by high-speed rotation, transmitting torque and bending stresses generated by its weight. Thus, the working condition of the supercritical unit is very poor, and the safety and reliability of the power plant is the most critical components in all parts of the power plant equipment. The rotor holds a large proportion of the generator. The structural design of the rotor is one of the keys to the development and design of the unit. At the same time, its manufacturing cost is high, difficult to retrofit. Once the rotor failure, the entire unit will be scrapped or paralysis[5,6,7]. Therefore, the finite element method is used to analyze the structure of the rotor at the beginning of the design. The strength analysis and the elasto-plastic analysis of the rotor and the wedges of the supercritical $1000 \mathrm{MW}$ turbo generator are especially important. 


\section{Strength analyses}

Establish the finite element model. In order to facilitate the study, the finite element model is simplified. Superimpose the centrifugal force of all coils and then apply a uniformly distributed load at the bottom of the wedges in contact with the coils. Establish a model only consisting of the rotor and wedges. The structure can be handled by plane, so we can select a cross-section of the rotor and the wedges for analysis and calculation. Establish a cross-sectional diagram of the rotor and the wedges shown in Fig. 1.

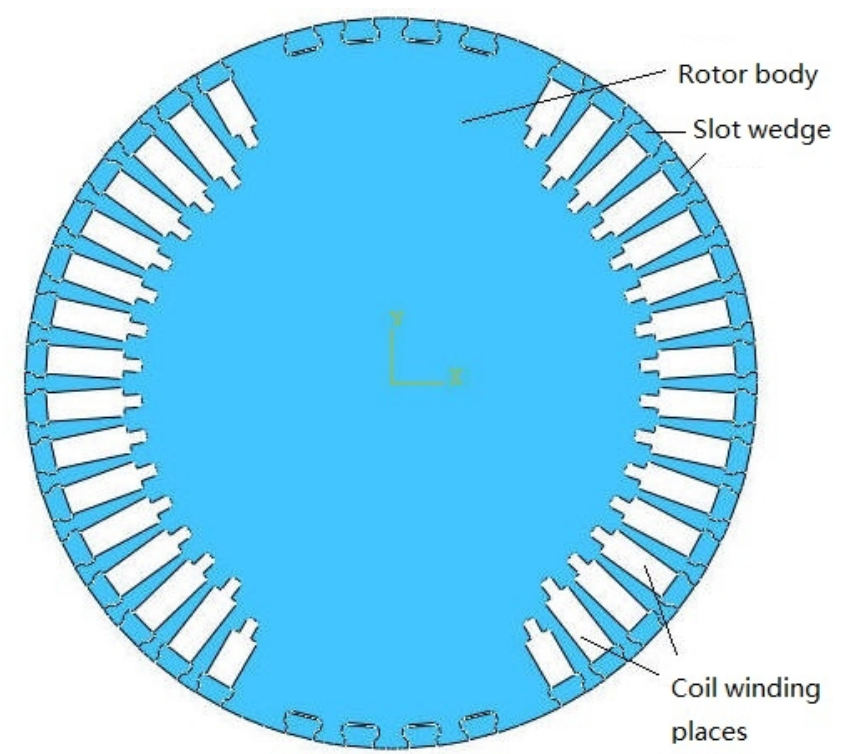

Fig. 1. Cross-sectional diagram of the rotor and the wedges

Treatment of finite element model. Firstly create the properties of materials and cross sections, and then enter the Property Module. Input the density, the elastic modulus and Poisson's ratio of material according to the data in Table 1 and define material properties of rotor and wedges respectively.

Table 1. Material properties

\begin{tabular}{ccccc}
\hline Parts & $\begin{array}{c}\text { Elastic Modulus } \\
\left(\mathrm{N} / \mathrm{mm}^{2}\right)\end{array}$ & $\begin{array}{c}\text { Poisson's } \\
\text { ratio }\end{array}$ & $\begin{array}{c}\text { Density } \\
\left(\mathrm{t} / \mathrm{mm}^{3}\right)\end{array}$ & $\begin{array}{c}\text { Yield limit } \\
(\mathrm{MPa})\end{array}$ \\
\hline Rotor & $E_{1}=2.1 E 5$ & $\mu=0.29$ & $\rho_{1}=7.85 E-9$ & $\sigma_{s 1}=690$ \\
\hline Aluminum wedge & $E_{1}=7.0 E 4$ & $\mu=0.30$ & $\rho_{1}=2.76 E-9$ & $\sigma_{s 1}=315$ \\
\hline $\begin{array}{c}\text { Anti-magnetic steel } \\
\text { wedge }\end{array}$ & $E_{1}=2.1 E 5$ & $\mu=0.29$ & $\rho_{1}=7.85 E-9$ & $\sigma_{s 1}=385$ \\
\hline
\end{tabular}

Secondly mesh into Mesh functional modules, set unit seed size of the rotor (or wedge) in each area and complete meshing set of rotor and wedge. Thirdly set the analysis step, enter the Step function module, the load is divided into 5 steps, so that the model can smooth transition. Then define contact, enter the Interaction function module, define the contact surface of the rotor and the slot wedge, select the two faces of the contact pair, set the relevant parameters, and complete the definition of contact. Last define the boundary and load, enter the Load function module, define the symmetry constraints in the $\mathrm{X}$ and $\mathrm{Y}$ directions for the rotor respectively, and apply load to the rotor and the wedge following the relevant steps, as shown in Fig. 2. 


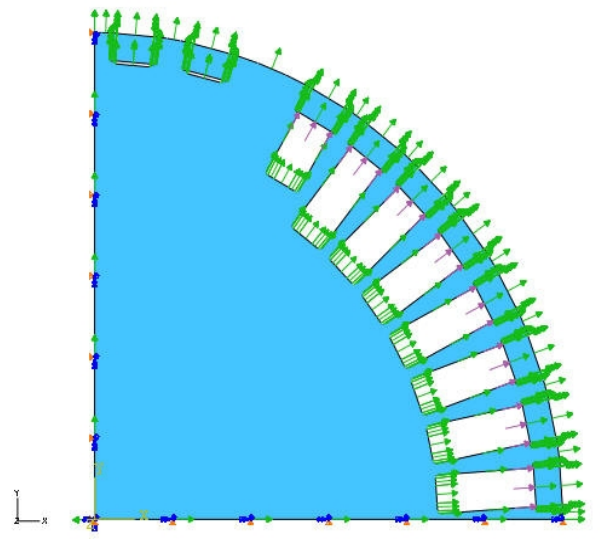

a) Aluminum wedge

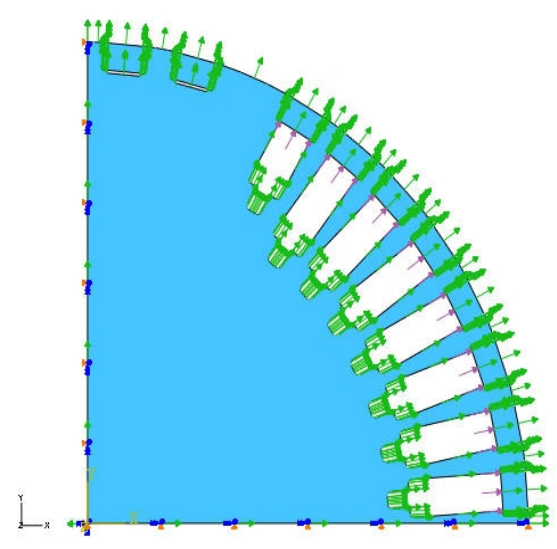

b) Anti-magnetic steel wedge

Fig. 2. Load application of the rotor and wedge

Results of finite element analysis. Enter into the Job function module, the stress distribution under different working conditions of the rotor and the wedge can be obtained through the strength analysis and calculation.

From the results after calculation and analysis, there are three points of the structure do not meet the strength design requirements, which are located in WAL2 chamfer and RAL3 chamfer of the aluminum wedge, and RST4 chamfer of the anti-magnetic steel wedge respectively.

\section{Elasto-plastic analysis}

The elastic and plastic analysis of the rotor and the wedge do not need to rebuild the model of the rotor and the wedge, and the original model can be modified. The main is to set the rotor and wedge of the elastic plastic properties and model load step.

Rotor and wedge material property settings. Enter into the Property function module, set the stress at the beginning of the plastic deformation of the rotor is 640MPa. Input data to complete the settings of the rotor material performance.

Rotor and wedge load step settings. Enter into the Load function module, select the Load Create, and apply load to the model as the following steps:

1) Input the rotating speed $\omega=10 \mathrm{rad} / \mathrm{s}$. Apply a small centrifugal force to the rotor and the wedge to make the model connect smoothly.

2) Change the rotating speed $\omega=100 \mathrm{rad} / \mathrm{s}$.

3) Apply pressure of coil to normal and abnormal wedge: $P_{\mathrm{n}}=P_{\mathrm{n}}^{\prime}=5 \mathrm{~N} / \mathrm{mm}^{2}$

4) Modify the coil pressure on the wedge: $P_{\mathrm{n}}=30 \mathrm{~N} / \mathrm{mm}^{2} P_{\mathrm{n}}^{\prime}=20 \mathrm{~N} / \mathrm{mm}^{2}$

5) Modify the coil pressure on the wedge: $P_{\mathrm{n}}=46 \mathrm{~N} / \mathrm{mm}^{2} \quad P_{\mathrm{n}}^{\prime}=29 \mathrm{~N} / \mathrm{mm}^{2}$ Change the rotating speed: $\omega=329 \mathrm{rad} / \mathrm{s}$ Apply all the elastic loads.

6) Elasto-plastic analysis of the rotor and the wedge model. Modify the pressure of the coil to the wedge: $P_{\mathrm{n}}=61.1 \mathrm{~N} / \mathrm{mm}^{2} P_{\mathrm{n}}^{\prime}=38.1 \mathrm{~N} / \mathrm{mm}^{2}$ Change the rotating speed: $\omega=377 \mathrm{rad} / \mathrm{s}$ Apply all loads. Load increment is set to 0.1 . Achieve incremental loading, and the geometric nonlinearity is set to ON.

Analysis results.After modifying the aluminum wedge portion of the rotor and the wedge model, enter into Job function module, and the stress distribution and plastic deformation zone of the rotor and wedge can be obtained after elasto-plastic analysis.

Fig. 3 shows the results of the elasto-plastic analysis about aluminum rotor. We can find that the maximum stress value of the rotor is $648.067 \mathrm{MPa}$ from Fig. 3(a). The plastic deformation area of the rotor is shown in Fig. 3 (b), with an area of about $0.25 \mathrm{~mm}^{2}$. The maximum plastic strain is 0.0022 . 


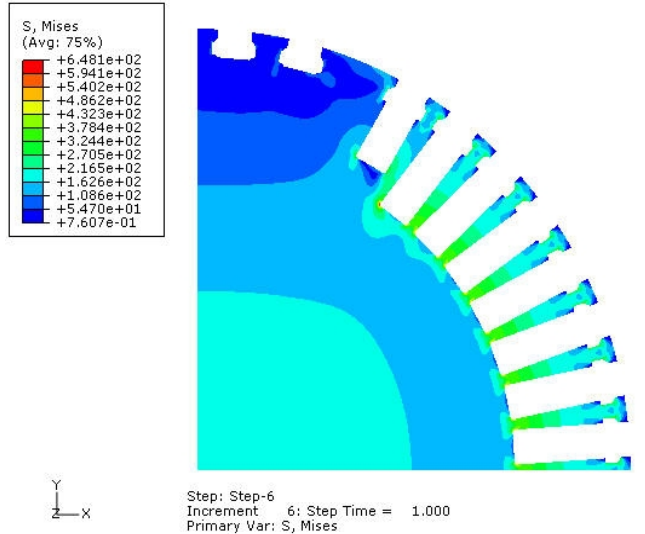

a) The stress distribution of the rotor

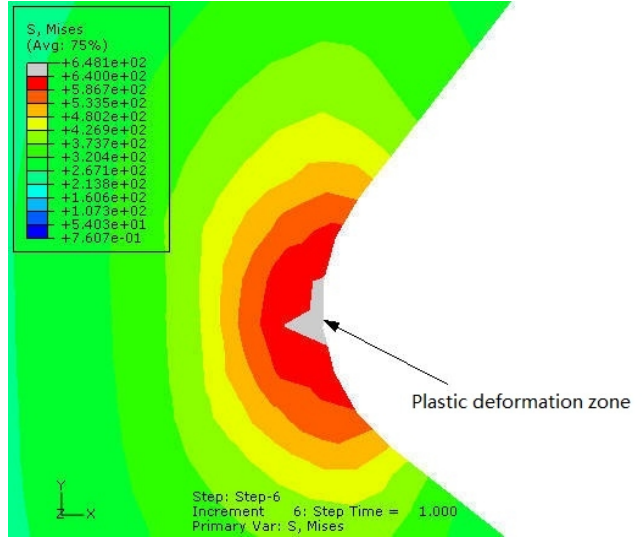

b) The plastic deformation area of the rotor

Fig. 3.The elasto-plastic analysis results of aluminum wedges of the rotor

Fig. 4 shows the results of the elasto-plastic analysis about aluminum wedge of the rotor. From Fig.4 (a) we can find that the maximum stress value of the wedge is $315.005 \mathrm{MPa}$ which does not reach the admissible stress value $339.926 \mathrm{MPa}$ obtained by strength analysis. The plastic deformation area of the rotor is shown in Fig. 4 (b), with an area of about $0.001 \mathrm{~mm}^{2}$. The maximum plastic strain is 0.0023 .

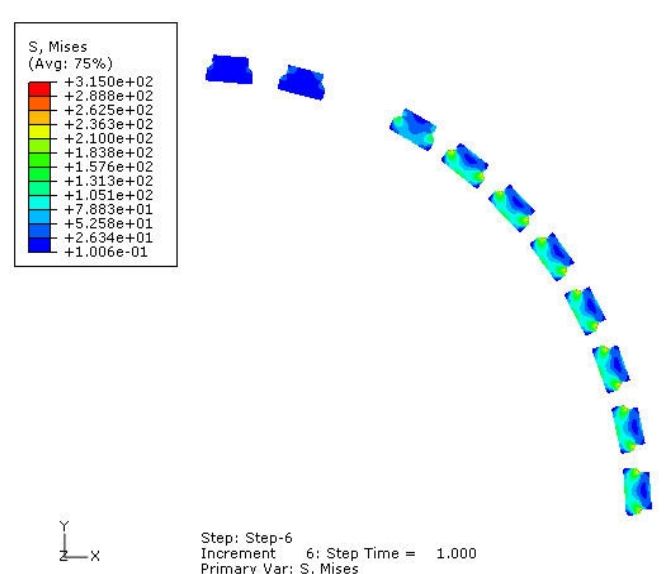

a) The stress distribution of aluminum wedges

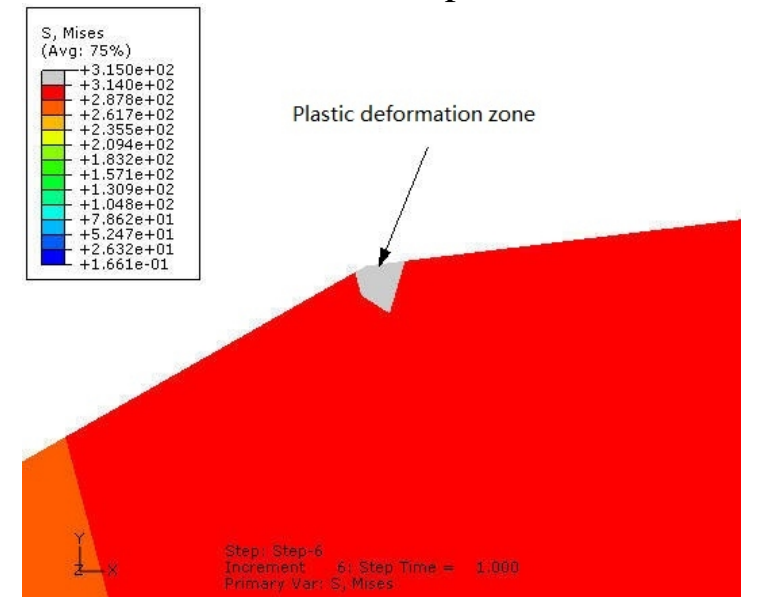

b) The plastic deformation area of the rotor

Fig. 4. The results of the elasto-plastic analysis of the aluminum wedges

Fig. 5 shows the results of the elasto-plastic analysis about anti-magnetic steel wedge of the rotor. From Fig. 5(a) we can find that the maximum stress value of the wedge is $643.695 \mathrm{MPa}$ which does not reach the admissible stress value $744.925 \mathrm{MPa}$ obtained by strength analysis. The plastic deformation area of the rotor is shown in Fig. 5 (b), with an area of about $0.66 \mathrm{~mm}^{2}$. The maximum plastic strain is 0.00094 .

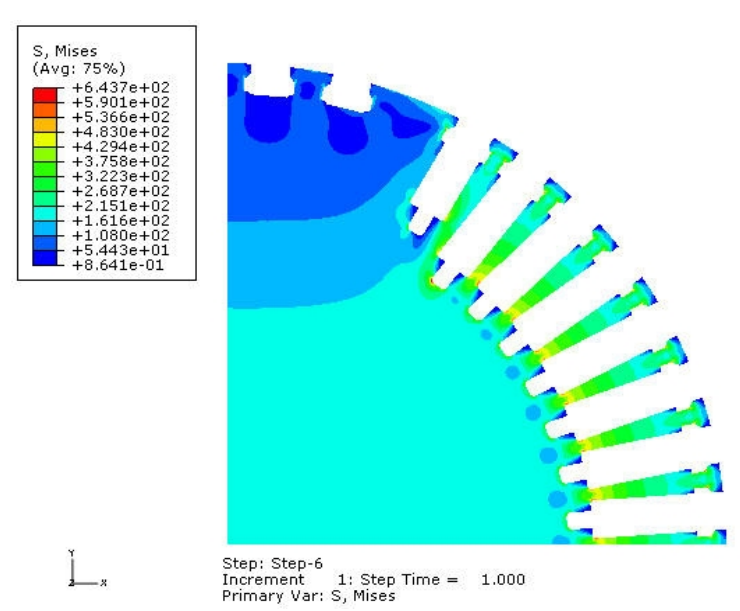

a) The stress distribution of the rotor

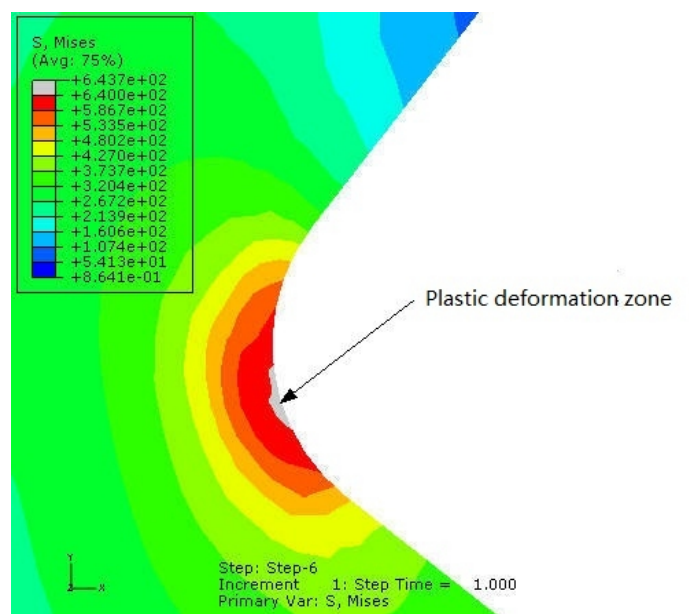

b) The plastic deformation area of the rotor

Fig. 5.The elasto-plastic analysis results of anti-magnetic steel wedge of the rotor 


\section{Best design}

When under normal working speed conditions, the safety factor at RAL3 chamfered of the aluminum wedges rotor is less than 1.2, the design does not meet the strength requirements. When under over speed condition, the RAL3 chamfered position of the aluminum wedge rotor, the WAL2 chamfer position of the aluminum wedge rotor and the RST4 chamfer position of the anti-magnetic steel wedge of the rotor occur yield, the design does not meet the strength requirements and needs to improve the structure

Aluminum wedge portion of the rotor structural improvements. Fillet radius at the RAL 3 chamfer position of the aluminum wedge portion of the rotor is $4 \mathrm{~mm}$, the stress gradients is large. In order to slow down the stress concentration, the chamfers radius are set to $5 \mathrm{~mm}, 6 \mathrm{~mm}, 7 \mathrm{~mm}, 8 \mathrm{~mm}$ and $9 \mathrm{~mm}$ respectively, then establish a finite element model and strength analysis: The rotor maximum stress value decreases with the increase of chamfer radius, increasing the fillet radius could significantly decrease the stress concentration. When the fillet radius increases to $8 \mathrm{~mm}$, the maximal rotor stress is $682.511 \mathrm{MPa}$ that is less than the yield strength of $690 \mathrm{MPa}$, which meets the design strength requirements. In addition, when the fillet radius is set to $8 \mathrm{~mm}$, the rotor stress maximum value under work speed conditions is $437.251 \mathrm{Mpa}$. The safety factor is 1.58 , which can meet the requirements of the design strength.

Improvement of aluminum slot wedge structure. The improvement of aluminum slot wedge structure has two ways without changing the wedge width: changing the aluminum wedge angle or changing the chamfer radius of the aluminum wedge at WAL2 chamfer position.

Influence of wedge angle on maximum stress value. The wedge angle of the slot wedge is set to $35^{\circ}, 40^{\circ}$ and $50^{\circ}$. Establish the finite element model and strength analysis. At different values of the wedge angle, the maximum stress value is $2.926 \mathrm{MPa}$ which accounts for $0.86 \%$ of total stress, and can be neglected. Therefore, the maximum stress values of the slot wedge are not significantly related to the wedge angle of the slot.

Influence of wedge fillet radius on the maximum stress value. Fillet radius of aluminum wedge at Wal2 chamfered position is set to $1.2 \mathrm{~mm} 、 1.6 \mathrm{~mm}$ and $2.0 \mathrm{~mm}$ respectively. Establish the finite element model and strength analysis. Wedges maximum stress decreases with increasing radius of the chamfer. When the fillet radius increased to $3 \mathrm{~mm}$, the maximum stress wedge reach $309.484 \mathrm{MPa}$ that is less than the yield limit $315 \mathrm{MPa}$, which meet the strength requirements.

Improved structure of anti-magnetic steel wedge of the rotor. Fillet radius of anti-magnetic steel wedge at RAL3 chamfered position is set to $7.2 \mathrm{~mm}, 8.0 \mathrm{~mm}$ and $8.8 \mathrm{~mm}$ respectively. Establish the finite element model and strength analysis. Rotor maximum stress value decreases with the increase of the radius of chamfer. When the fillet radius increased to $8.0 \mathrm{~mm}$, the maximum rotor stress reach $675.016 \mathrm{MPa}$ that is less than the yield limit $690 \mathrm{MPa}$, which meet the strength requirements.

\section{Conclusions}

In this paper, we extracted and simplified physical model of the rotor and the wedge. Establish the finite element model and analysis the strength of the rotor and the wedge by software ABAQUS, then obtain the stress distributions of the rotor and the wedge under two different conditions. The plastic deformation area and plastic strain of the rotor and the slot wedge under the over speed condition were obtained through the model elastoplastic analysis. By comparing the maximum stress values of the rotor and slot wedge with different structural parameters under the same working condition, the optimum design of the rotor and slot wedge and several conclusions were obtained as follows:

1. Establish the finite element model and analysis the strength of the rotor and the wedge by software ABAQUS, then obtain the stress distributions of the rotor and the wedge under two different conditions. The chamfer strength at tooth root and shoulder end of the aluminum wedge and sub slot chamfer of anti-magnetic steel did not meet the design strength requirements.

2. The actual stress distribution, plastic deformation area and maximum plastic strain of the rotor and the slot wedge under the over speed working conditions were obtained by the elastic plastic 
analysis. The maximum stress value of aluminum wedge is $648.067 \mathrm{MPa}$, and plastic deformation area is $0.25 \mathrm{~mm}^{2}$; the maximum stress value of anti-magnetic wedge is $643.695 \mathrm{MPa}$, plastic deformation area is $0.66 \mathrm{~mm}^{2}$.In contrast, the maximum stress value of the aluminum wedge is large, but the plastic deformation area is small, which is caused by the different stress gradient. The fillet radius at the dangerous point of the aluminum wedge rotor is $4 \mathrm{~mm}$; the stress gradient is large. The fillet radius at the dangerous point of the anti-magnet rotor is $6.4 \mathrm{~mm}$; the stress gradient is small.

3. For the dangerous point of the rotor, chose several groups of different parameters for strength analysis. Comparing the results, a conclusion can be drawn that the maximum stress value of the rotor decreases with increase of the chamfer radius and increasing the fillet radius can mitigate the stress concentration significantly.

4. The average pressure of the slot wedge is not related to the wedge angle of the slot. The maximum stress value of the slot wedge is not significantly related to the wedge angle through the analysis and calculation to the model with different slot wedge angle.

5. Select and analysis different radius of curvature at the corner of the slot. The maximum stress value is significantly increased with the decrease of the radius of curvature at the corner of the slot wedge by comparing the results of finite element analysis.

In summary, the finite element analysis of the large turbo generator rotor and slot wedge using ABAQUS software is feasible, and the analysis results are basically consistent with the actual situation. The finite element method can also be used in the static analysis and dynamic analysis of other large and complex parts.

\section{References}

[1] K Wang. Research on the life of large steam turbine rotor [D]. Wu Han: Huazhong University of Science and Technology, 2004.

[2] Z.P. Song. China Engineering Science2002, 2: 22-44.

[3] G Wang, Ximing Li. Design, manufacture and operation of large steam turbine generator[M]. Shanghai: Shanghai science and Technology Publishing House, 2000.

[4] N.S. Hu. Steam turbine equipment and systems[M].Beijing: China Electric Power Press,2006.

[5] S.H. Huang. Journal of Power and Energy, 1993, 207(49): 49-53.

[6] V N Shlyannikov, B.V. Iltchenko, N.V. Stepanov. Engineering Failure Analysis, 2001, 8: 461-475.

[7] W.T. Fu, B.Z. Zhang, B.Z. Wang. Heavy Castings and Forgings, 2008(5):33-41. 